\title{
Electronic structure of $A_{4} \mathrm{C}_{60}$ : Joint effect of electron correlation and vibronic interactions
}

\author{
L. F. Chibotaru, A. Ceulemans, and S. P. Cojocaru* \\ Division of Quantum Chemistry, University of Leuven, Celestijnenlaan 200F, B-3001 Leuven, Belgium
}

(Received 26 February 1999)

\begin{abstract}
Effects of electron correlation, intrasite vibronic interaction, and merohedral disorder on the electronic structure of $\mathrm{K}_{4} \mathrm{C}_{60}$ are investigated with a model approach taking into account all essential interactions in the lowest unoccupied molecular orbital (LUMO) band. The self-energy was calculated within the $G W$ approximation with self-consistency after the quasiparticle Green function starting from Hartree-Fock band structure. The insulating state arises due to interorbital charge disproportionation within the LUMO band while the band gap is strongly reduced by effects of long-range electron correlation. The results of the calculations are in reasonable agreement with experiment, providing evidence for a Jahn-Teller induced transition from a MottHubbard to a band insulator state. [S0163-1829(99)51020-5]
\end{abstract}

The electronic structure of alkali-doped fullerides $A_{4} \mathrm{C}_{60}$ ( $A=\mathrm{K}, \mathrm{Rb}, \mathrm{Cs})$ is markedly different from similar compounds, $A_{3} \mathrm{C}_{60}$. Indeed, while $A_{3} \mathrm{C}_{60}$ are metals and even superconductors $^{1,2}$ well described by the Fermi-liquid theory, ${ }^{3} A_{4} \mathrm{C}_{60}$ are insulators. ${ }^{4-6}$ Band-structure calculations of local-density approximation (LDA) and extended Hückel type $^{2,7,8}$ have shown that the opening of the insulating gap cannot be explained by the tetragonal splitting of the threefold degenerate lowest unoccupied molecular orbital (LUMO) orbitals in the body-centered-tetragonal (bct) lattice of these materials.

On the other hand, there is a body of evidence coming from both experiment and calculations that the electron correlation should be strong in fullerides ${ }^{1}$ making the MottHubbard scenario for the insulating state of $A_{4} \mathrm{C}_{60}$ quite plausible. Moreover, the estimations gave the values of $1-1.5 \mathrm{eV}$ for the intrasite electron repulsion $(U)$ and $0.5-0.6 \mathrm{eV}$ for the LUMO bandwidth $(w)$ indicating thus that all stoichiometric compounds with partly filled $t_{1 u}$ orbitals on fullerene sites should be Mott-Hubbard insulators. The calculations performed within the simple Hubbard model with degenerate sites $^{9,10}$ revealed, however, an important qualitative trend. It was found that the critical value of the ratio $U / w$ at which the Mott-Hubbard transition takes place increases with orbital degeneracy of sites due to a corresponding increase of the scattering space for electrons. ${ }^{10}$ All cubic fullerides are thus expected to be near the border of this transition. It was also pointed out that the critical value of $U / w$ depends on the type of the crystal lattice being higher in frustrated lattices. ${ }^{11}$ This naturally places fcc $A_{3} \mathrm{C}_{60}$ and bct $A_{4} \mathrm{C}_{60}$ on different sides of the transition despite close values of $U$ and $w$ in both compounds. Finally the absence of the antiferromagnetic ordering, expected in the bipartite lattice of $A_{4} \mathrm{C}_{60}$, was explained by non-negligible next-nearest-neighbor electron transfer present in these compounds. ${ }^{12}$

An important interaction not included in the above consideration is the Jahn-Teller effect within the $t_{1 u}$ shell on fullerene sites. It was shown previously by simple band calculations ${ }^{13}$ that the concomitant account of the intrasite bielectronic and vibronic interactions can lead to an electronic instability characterized by interorbital disproportionation of the LUMO charge density at each site. For the case of threefold degenerate sites, such instability arises when the condition $6 E_{J T}>7 J$ is fulfilled, where $E_{J T}$ is the energy of Jahn-Teller stabilization of the sites populated by a single electron and $J$ is the parameter of the intrasite exchange interaction. In contrast to conventional band-structure calculations, ${ }^{2}$ the interorbital charge disproportionation effect results in a pronounced nonrigid behavior of the LUMO band on doping, ${ }^{13}$ in full agreement with the experiment. ${ }^{14,15}$ In particular, it predicts a strongly enlarged metallic LUMO band for $A_{3} \mathrm{C}_{60}$ and an insulating state for $A_{4} \mathrm{C}_{60}$ with similar electronic parameters, which is also in line with experiment. For $A_{3} \mathrm{C}_{60}$ compounds, more detailed calculations were able to reproduce the maximum of the density of states (DOS) at the Fermi level ${ }^{16}$ and specific features in the optical conductivity spectra ${ }^{17,18}$ assigned as transitions between disproportionated subbands of the LUMO band. ${ }^{19}$

In fact, the Mott-Hubbard and the orbital disproportionation pictures are two different limiting cases with respect to the involvement of vibronic interactions. Indeed, the first mechanism supports an equal participation of the orbital components of each fullerene site in the ground state ${ }^{10}$ leading to their equal population, and in turn, to zero values of equilibrium Jahn-Teller distortions. At the same time these distortions represent the fingerprint of the second mechanism. ${ }^{13}$ Unfortunately the existence of these distortions cannot be tested directly in x-ray diffraction because of merohedral disorder of fullerene molecules in the compounds with intermediate doping $(0<n<6)$. Indirect evidence for Jahn-Teller distortions has been found so far only in $A_{4} \mathrm{C}_{60}$. This is suggested by the splitting of the $T_{1 u}(4)$ vibrational mode ${ }^{17}$ and the characteristic structure of the $t_{1 u} \rightarrow t_{1 g}$ absorption band. ${ }^{20,21}$ It seems therefore justified to describe the ground state and the low-lying electronic excitations in these compounds starting from the orbital disproportionation limit.

The Hamiltonian for the LUMO band includes transfer, bielectronic, and vibronic contributions. ${ }^{13,16}$ The transfer part describes the $t_{1 u}-t_{1 u}$ electron transfer between nearest and two distinct next-nearest neighbors for two standard orientations of each fullerene molecule simulating the merohedral disorder. $^{22}$ The hopping amplitudes have been obtained using a recent tight-binding parametrization for $\mathrm{K}_{4} \mathrm{C}_{60}$ that repro- 
duces satisfactorily the LDA LUMO band. ${ }^{12}$ The bielectronic part includes the intrasite electron repulsion, $U_{0}=2.7 \mathrm{eV}$, (Refs. 1 and 23), the intersite bare Coulomb repulsion, and the intrasite exchange interaction, $J=0.05 \mathrm{eV}$. The vibronic part consists of the interaction with on-site nuclear distortions of $H_{g}$ type. In the static approximation the nuclear coordinates can be eliminated by expressing them through the elements of the one-particle density matrix of the corresponding sites. ${ }^{13}$ The effect of these distortions is thus expressed by a single parameter, the Jahn-Teller energy, estimated as $E_{J T}=0.1 \mathrm{eV} .{ }^{1}$ Other bands, not included explicitly, contribute to the screening of the bielectronic interactions within the LUMO band, which can be treated in our case only by a model description of the dielectric constant $\epsilon(q, \omega)$. Starting with the experimental $\omega$ dependence of the dielectric constant, the $q$ dependence of its real part was obtained by applying the model treatment of Gunnarsson et al. ${ }^{24}$ Following this approach, the molecular polarizability function, $\alpha(q)$, was simulated by replacing the $\mathrm{C}_{60}$ molecules by metallic spheres. Then the response of $\mathrm{C}_{60}$ molecules on the bct lattice corresponding to $\mathrm{K}_{4} \mathrm{C}_{60}[a=11.886$ $\AA, c=10.774 \AA$ (Ref. 21)] to an external q-dependent electric field was found within the Lorentz-Lorentz approach.

First we consider a Hubbard-type model retaining only the intrasite part of the bielectronic interactions. The Hubbard parameter, $U$, is defined as the intrasite repulsion of two electrons screened by the electron excitations from all occupied bands except for the LUMO band. It was derived from the inverse Fourier transformation of $U(q) / \operatorname{Re}[\epsilon(q, 0)]$ within the first Brillouin zone; here $U(q)$ is the Fourier transform of the Coulomb repulsion in the site representation and $\epsilon$ is the dielectric constant of the bct lattice of neutral $\mathrm{C}_{60}$ 's $\left[\alpha(0)=85 \AA^{3}\right]$. In this way one obtains $U=1.24 \mathrm{eV}$, which is in good agreement with previous estimations. ${ }^{1,21}$ The results of the Hartree-Fock calculations for this model are shown in Fig. 1. The dashed line shows the first step of the calculations corresponding to conventional [i.e., to the LDA (Ref. 12)] $t_{1 u}$ DOS for an ordered lattice. ${ }^{25}$ A nonequal population of three $t_{1 u}$ orbitals at each site arises already at this step, $n_{x}=1.41, n_{y}=1.10, n_{z}=1.49$, as a consequence of the transfer anisotropy induced by the bct lattice. At the selfconsistency point the band structure is resolved into fully disproportionated subbands, $n_{x}=2, n_{y}=0, n_{z}=2$ (full line in Fig. 1). Inclusion of the merohedral disorder does not introduce new qualitative features in the picture (dotted versus chain lines in Fig. 1). It should be stressed that unlike cubic crystals, where disproportionation can arise only as broken symmetry solution for band orbitals ${ }^{13}$ the present treatment is within the conventional Hartree-Fock frame. The obtained band structure corresponds therefore to a band insulator although not a conventional one because of the large bielectronic contribution to the insulating gap. The relative shift of the disproportionated subbands is described by the parameter $U_{d}=U+6 E_{J T}-5 J,{ }^{16}$ which amounts to $1.6 \mathrm{eV}$ in our case. The occupied subbands in Fig. 1 are obviously too narrow while the insulating gap is obtained too large $(1.1 \mathrm{eV})$ as compared to the experiment. The latter arises from the unscreened exchange interaction used in Hartree-Fock calculations. As a result, the self-interaction part, $U$, makes the main contribution to $U_{d}$.

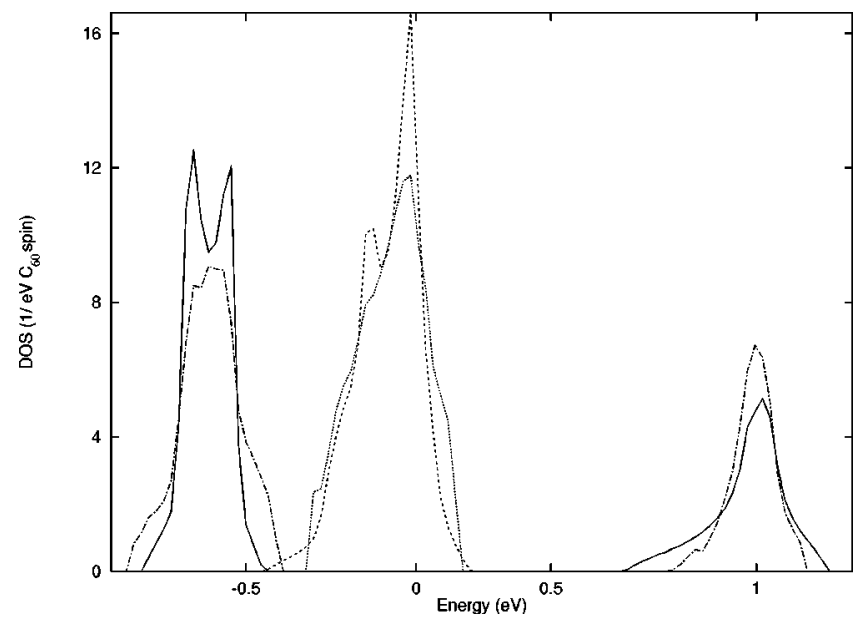

FIG. 1. Calculated density of states for the $t_{1 u}$ LUMO band within the Hartree-Fock approximation; dashed and solid lines correspond, respectively, to equal electron population of the three orbital components at each site, and to the self-consistency point (full disproportionation) for an orientationally ordered crystal; similarly dotted and chained lines describe the case of merohedral disorder simulated here by a random distribution of two standard orientations of fullerene molecules (Ref. 22) within an enlarged unit cell containing 250 sites. The zero of energy is chosen at the Fermilevel position of the nondisproportionated band structure of an ordered crystal.

A proper description of the one-particle excitations within the obtained band structure requires electron correlation that is introduced here within the $G W$ approximation. ${ }^{26}$ This approximation turned out to be especially successful for band insulators ${ }^{27}$ and hence might be appropriate in our case also. The electron self-energy is conveniently represented in this method as a sum of two physically distinct contributions, the screened exchange, $\Sigma^{s x}$, and the Coulomb hole, $\Sigma^{c h}$, parts. The quasiparticle energies for a given band state $|\alpha \mathbf{k}\rangle$ are approximated through diagonal matrix elements of the selfenergy:

$$
E_{\alpha \mathbf{k}}=\tilde{\varepsilon}_{\alpha \mathbf{k}}+\operatorname{Re} \Sigma_{\alpha}^{s x}\left(\mathbf{k}, E_{\alpha \mathbf{k}}\right)+\operatorname{Re} \Sigma_{\alpha}^{c h}\left(\mathbf{k}, E_{\alpha \mathbf{k}}\right)
$$

In Eq.(1), $\tilde{\varepsilon}_{\alpha \mathbf{k}}$ is the band energy calculated within the Hartree approximation. In order to have a better starting point one may use LDA wave functions and energies for the band states. In this case $\widetilde{\varepsilon}_{\alpha \mathbf{k}}$ would correspond to the LDA orbital energy corrected by the effect that the exchange-correlation potential has on this state. ${ }^{27}$ In our case the deviation from the Hartree potential is due to the subtraction of the selfinteraction, therefore,

$$
\widetilde{\varepsilon}_{\alpha \mathbf{k}}=\varepsilon_{\alpha \mathbf{k}}+\left\langle\alpha \mathbf{k}\left|U_{s-i}\right| \alpha \mathbf{k}\right\rangle,
$$

where $\varepsilon_{\alpha \mathbf{k}}$ and $|\alpha \mathbf{k}\rangle$ are orbital energies and wave functions describing the disproportionated subbands in Fig. 1. $U_{s-i}$ is the operator of the electron self-interaction:

$$
U_{s-i}=U \sum_{\mathbf{n} i i^{\prime} \sigma} D_{\mathbf{n} i^{\prime} \sigma}^{\mathbf{n} i \sigma} a_{\mathbf{n} i^{\prime} \sigma}^{\dagger} a_{\mathbf{n} i \sigma}
$$


where $D_{\mathbf{n} i^{\prime} \sigma}^{\mathbf{n} i \sigma}$ is the element of the one-particle density matrix and $i, i^{\prime}$ denote the components of the orbital triplet $t_{1 u}$ at each site $\mathbf{n}$. Using these notations one can write for the components of the self-energy,

$$
\begin{aligned}
\operatorname{Re} \sum_{\alpha}^{s x}(\mathbf{k}, \omega)=- & \sum_{\mathbf{k}^{\prime} \alpha^{\prime}}^{o c c} U_{\alpha \alpha^{\prime}}^{\alpha^{\prime} \alpha}\left(\mathbf{k}-\mathbf{k}^{\prime}\right) \operatorname{Re} \frac{1}{\epsilon\left(\mathbf{k}-\mathbf{k}^{\prime}, \omega-\tilde{\varepsilon}_{\mathbf{k}^{\prime} \alpha^{\prime}}\right)}, \\
\operatorname{Re} \Sigma_{\alpha}^{c h}(\mathbf{k}, \omega)= & -\sum_{\mathbf{k}^{\prime} \alpha^{\prime}}^{a l l} U_{\alpha \alpha^{\prime}}^{\alpha^{\prime} \alpha}\left(\mathbf{k}-\mathbf{k}^{\prime}\right) \\
& \times \frac{1}{\pi} \mathrm{P} \int_{0}^{\infty} \frac{\operatorname{Im}\left[1 / \epsilon\left(\mathbf{k}-\mathbf{k}^{\prime}, \omega^{\prime}\right)\right]}{\omega-\omega^{\prime}-\widetilde{\varepsilon}_{\mathbf{k}^{\prime} \alpha^{\prime}}} d \omega^{\prime},
\end{aligned}
$$

where $U_{\alpha \alpha^{\prime}}^{\alpha^{\prime} \alpha}\left(\mathbf{k}-\mathbf{k}^{\prime}\right)$ is the exchange integral corresponding to the band orbitals $|\alpha \mathbf{k}\rangle$ and $\left|\alpha^{\prime} \mathbf{k}^{\prime}\right\rangle$.

The dielectric function was derived from electron-energyloss spectroscopy (EELS) data as follows. The loss function for $\mathrm{K}_{4} \mathrm{C}_{60}$ available for the low-energy region (up to $2.5 \mathrm{eV}$ ) at momentum transfer $q_{0}=0.15 \AA^{-1}$ (Ref. 20) was combined with the loss function for $\mathrm{C}_{60}$ at the same momentum transfer. $^{28} \operatorname{Re}\left[1 / \epsilon\left(q_{0}, \omega\right)\right]$ was then obtained via the Kramers-Kronig transformation and the dielectric function was found at the given value of $q_{0}$ via the inversion of $1 / \epsilon$. The quality was checked by reproducing the optical conductivity data for $\mathrm{K}_{4} \mathrm{C}_{60}$ in Ref. 21 . These are also in good agreement with direct optical measurements, ${ }^{17}$ which validates the inversion procedure for the dielectric matrix equivalent to the neglect of the local-field effects. ${ }^{29}$ For each $\operatorname{Re}\left[\epsilon\left(q_{0}, \omega\right)\right]$ an effective site polarizability was obtained and then the $q$ dependence of the real part was extended over the twice enlarged Brillouin zone using the Lorentz-Lorentz approach as it was described above. After the second Kramers-Kronig transformation, $\operatorname{Im}[\epsilon(q, \omega)]$ was found and, finally, the quantities entering Eqs. (4) were derived.

Figure 2 illustrates the outcome of our calculations for the quasiparticle DOS of an orientationally ordered compound. The dashed line corresponds to the first iteration when the quasiparticle energy equation (1) is solved using Eqs. (4), i.e., with the noninteracting Green function in the self-energy expressions. This approximation $\left(G_{0} W\right)$ is usually sufficient for a reasonable description of the one-electron excitations in many semiconductors and insulators. When it is not the case a self-consistency after the Green function is required. It is known, however, that the full self-consistency in the sense of Dyson equation leads to wrong results. ${ }^{27}$ Recently a restricted version based on the self-consistent determination of a nonlocal potential added to $G_{0}$ in order to simulate the effect of self-energy corrections was proposed. ${ }^{30}$ This permits us to take into account the modification of the oneelectron wave functions with the band gap. Such an effect is unimportant for $A_{4} \mathrm{C}_{60}$ because of a very small hybridization of disproportionated subbands when the insulating gap opens. We used therefore another version by extending the self-consistency over the real part of the self-energy in $G$. This corresponds to the replacement of the band energies $\tilde{\varepsilon}_{\mathbf{k} \alpha}$ by quasiparticle energies $E_{\mathbf{k} \alpha}$ in the self-energy expressions (4). The result of such a restricted self-consistency for the

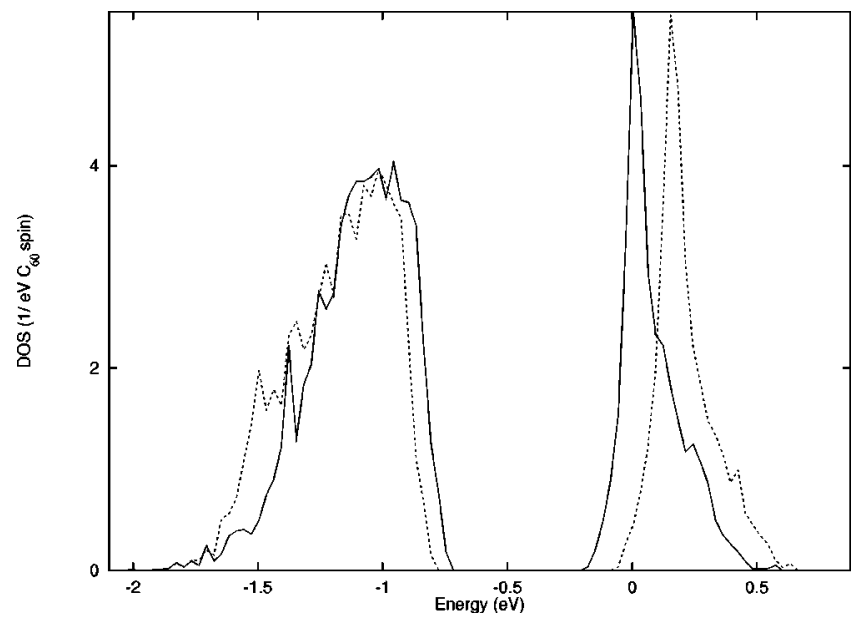

FIG. 2. The quasiparticle density of states for the $t_{1 u}$ LUMO band calculated within the $G W$ approximation in an ordered crystal; the dashed line corresponds to the first iteration $\left(G_{0} W\right)$ and the solid line corresponds to a restricted self-consistency after the quasiparticle Green function. The sharp features in the lowest band reflect the noise level of the calculations.

quasiparticle DOS is shown in Fig. 2 by the solid line. We can see that the occupied bands become strongly enlarged as compared with the result in Fig. 1 approaching the value of $0.7 \mathrm{eV}$ for the full width at half maximum seen in photoemission. ${ }^{31}$ The proper description of the quasiparticle DOS would require the consideration of the imaginary part of the self-energy that leads to the reduction of the quasiparticle weight and to the incoherent transfer of the spectral weight resulting in further enlargement of the bands. The inclusion of the merohedral disorder will also contribute to the modification of the DOS profile. We believe, however, that these effects will not affect our conclusions. An important result of the calculations in Fig. 2 is the appreciable reduction of the insulating gap $(0.5 \mathrm{eV})$ which agrees well with EELS data (0.3-0.4 eV) (Refs. 20 and 21), but is still large as compared with optical and other measurments (0.1$0.2 \mathrm{eV}){ }^{17,32,33}$ The inspection of the dispersion curves of the self-energy components shows that the main contribution to the insulating gap comes from the screened exchange part of the self-energy. This is strongly influenced by the lowfrequency part of the dielectric function, dominated by the excitations from the partly filled $t_{1 u}$ shell. Since the dielectric function was constructed from EELS the obtained gap is consistent with these data. The main improvement in the quantitative description of the gap is expected to be achieved through a better description of the dielectric function in the low-energy region. The uncertainities in the parameters $E_{J T}$ and $J$ can also modify the gap by $0.1-0.2 \mathrm{eV}$.

We have thus shown that the observed picture of oneparticle excitations in $A_{4} \mathrm{C}_{60}$ is fully consistent with the orbital disproportionation scenario. This mechanism represents a natural way the Jahn-Teller distortions can arise in crystals with strong electron correlation. The resulting insulating state, however, is quite different from that of a MottHubbard insulator because it is described predominantly by a single determinant constructed from occupied band states. In this respect the picture is close to a band insulator. Unlike the conventional band insulators, in the case of orbital disproportionation the gap arises mainly due to bielectronic interactions. These can efficiently be screened by long-range 
electron correlations, which results in a small insulating gap as the data for $A_{4} \mathrm{C}_{60}$ clearly show. In conclusion the study of these systems has revealed the presence of a new mechanism to change a Mott-Hubbard insulator into a band insulator through a Jahn-Teller effect.
We are grateful to O. Gunnarsson for stimulating discussions, and thank M. Knupfer for sending us the preprint of Ref. 21 prior to publication. The financial support of the Flemish Government (Ministerie voor Programmatie van het Wetenschapsbeleid) is acknowledged.
*On leave from the Institute of Applied Physics, Academy of Sciences, Kishinev, Moldova.

${ }^{1}$ O. Gunnarsson, Rev. Mod. Phys. 69, 575 (1997).

${ }^{2}$ M.P. Gelfand, Supercond. Rev. 1, 103 (1994).

${ }^{3}$ T.T.M. Palstra et al., Phys. Rev. B 50, 3462 (1994).

${ }^{4}$ D.W. Murphy et al., J. Phys. Chem. Solids 53, 1321 (1992).

${ }^{5}$ R.F. Kiefl et al., Phys. Rev. Lett. 69, 2005 (1992).

${ }^{6}$ P.J. Benning et al., Phys. Rev. B 47, 13843 (1993).

${ }^{7}$ R.C. Haddon, Acc. Chem. Res. 25, 127 (1992).

${ }^{8}$ S.C. Erwin and C. Bruder, Physica B 199-200, 600 (1994).

${ }^{9}$ J.P. Lu, Phys. Rev. B 49, 5687 (1994).

${ }^{10}$ O. Gunnarsson, E. Koch, and R.M. Martin, Phys. Rev. B 54, R11 026 (1996).

${ }^{11}$ O. Gunnarsson, E. Koch, and R.M. Martin, Phys. Rev. B 56, 1146 (1997); in Electronic Properties of Novel Materials: Progress in Molecular Nonostructures, Proceedings of the XII International Winterschool, AIP Conf. Proc. No. 442, edited by H. Kuzmany et al. (AIP, New York, 1998), p. 287.

${ }^{12}$ O. Gunnarsson et al., Phys. Rev. B 57, 2159 (1998).

${ }^{13}$ L.F. Chibotaru and A. Ceulemans, Phys. Rev. B 53, 15522 (1996).

${ }^{14}$ M.S. Golden et al., J. Phys.: Condens. Matter 7, 8219 (1995).

${ }^{15}$ J.H. Weaver, Acc. Chem. Res. 25, 143 (1992).

${ }^{16}$ A. Ceulemans, L.F. Chibotaru, and F. Cimpoesu, Phys. Rev. Lett. 78, 3725 (1997).

${ }^{17}$ Y. Iwasa and T. Kaneyasu, Phys. Rev. B 51, 3678 (1995).

${ }^{18}$ L. Degiorgi et al., Phys. Rev. B 49, 7012 (1994).
${ }^{19}$ L.F. Chibotaru and A. Ceulemans, in Proceedings of the XIV International Symposium on Jahn-Teller Effect, edited by G. Bevilacqua, L. Martinelli, and N. Terzi (World Scientific, Singapore, in press).

${ }^{20}$ M. Knupfer, J. Fink, and J.F. Armbruster, Z. Phys. B 101, 57 (1996).

${ }^{21}$ M. Knupfer and J. Fink, Phys. Rev. Lett. 79, 2714 (1997).

${ }^{22}$ T. Yildirim et al., Phys. Rev. B 48, 12262 (1993).

${ }^{23} U_{0}$ describes the bare (i.e., not screened by surrounding molecules) effective Coulomb repulsion of two electrons in the same $t_{1 u}$ orbital.

${ }^{24}$ O. Gunnarsson et al., J. Phys.: Condens. Matter 8, 2557 (1996).

${ }^{25}$ The orientational order was described in the present calculations by a standard orientation A of each molecule (Ref. 22).

${ }^{26}$ L. Hedin, Phys. Rev. 139, A796 (1965); L. Hedin and S. Lundqvist, in Solid State Physics, edited by H. Ehrenreich, F. Seitz, and D. Turnbull (Academic, New York, 1969), Vol. 23, p. 1.

${ }^{27}$ F. Aryasetiawan and O. Gunnarsson, Rep. Prog. Phys. 61, 237 (1998).

${ }^{28}$ E. Sohmen and J. Fink, Phys. Rev. B 47, 14532 (1993).

${ }^{29}$ M.S. Hybertsen and S.G. Louie, Phys. Rev. B 34, 5390 (1986).

${ }^{30}$ F. Aryasetiawan and O. Gunnarsson, Phys. Rev. Lett. 74, 3221 (1995).

${ }^{31}$ M. De Seta and F. Evangelisti, Phys. Rev. B 51, 1096 (1995).

${ }^{32}$ G. Ruani et al., Physica C 235-240, 2477 (1994).

${ }^{33}$ R. Kerkoud et al., J. Phys. Chem. Solids 57, 143 (1996). 